\title{
Demystify the Insidious Role of Lumbar Disc Degeneration In Low Back Pain
}

\section{TO THE EDITOR:}

I read with great interest and excitement the article by Chang-Jiang Zheng and James Chen, entitled "Disc Degeneration Implies Low Back Pain" published in Theoretical Biology and Medical Modelling (1). Zheng and Chen used a system dynamics approach to build a physiologically based model investigating the relationship between disc degeneration and low back pain. They found that a three-compartment model (no disc degeneration, disc degeneration with pain remission, disc degeneration with pain recurrence) accurately predicts the age-specific prevalence observed in one of the largest population-based surveys ( $R 2=0.998)$. The model also fully accounts for the age-specific prevalence of disc degeneration detected with lumbar magnetic resonance imaging (MRI) among asymptomatic individuals $(R 2=0.978)$. Zheng and Chen conclude that disc degeneration is the driving force behind and the cause of age dependence in low back pain, and the observed complexity of low back pain epidemiology arises from the slow dynamics of disc degeneration coupled with the fast dynamics of disease recurrence (1).

I would like to commend Zheng and Chen for their novel and assiduous work in bringing to fruition a mechanistic explanation for the asymptomatic disc degeneration conundrum and proposing that disc degeneration is the predominant cause of low back pain. Unquestionably, this is of seminal significance because of the existence of this long-standing mystery among clinicians (even pain management physicians), patients, media, health care policy makers, and insurance carriers about whether disc degeneration is part of the "normal aging process" that should not be painful, since it can frequently be found in many asymptomatic patients $(2,3)$.

Low back pain is a leading cause of disability worldwide and the second most common cause of physician visits (4). Recently, Vos and colleagues (5) published a mega study, entitled "Global, Regional, and National Incidence, Prevalence, and Years Lived with Disability for 301 Acute and Chronic Diseases and Injuries in 188 Countries, 1990-2013: A Systematic Analysis for the Global Burden of Disease Study 2013," in the Lancet. Of 301 acute and chronic diseases and injuries in 188 countries, low back pain ranks first among the top 10 leading causes of YLDs (years lived with disability). Low back pain exerts a tremendous disease burden on individual patients and society at a global level, due to its prevalence and ability to cause long-term disability.

Surprisingly, I happen to have a personal story to share, as a patient with low back pain. My story goes like this: My low back pain started insidiously in early 1996 without any preceding event. I initially thought it would go away on its own within 6 to 8 weeks, like most of the medical text books taught, but it did not. As a physician by training, I took advantage of all available resources online, tried all recommended treatment modalities, in vain. My back pain was relentless and progressive. On August 28, 1996, I took my United States Medical Licensing Exam (USMLE) Step II at Boston testing center. Upon finishing the exam, I could barely stand up. I managed to, but I could not straighten my back. It felt like the pain could cause my back to "collapse" at any second. While leaving the test center to meet a fellow medical graduate friend who was waiting for me in the hallway, I was having a very difficult time. Seeing me walking like a 90-year-old, he was in disbelief. Because 10 years prior when we were both medical students in China, I was the most fit, energetic, and a well-known athlete (sprinter, soccer player) on campus. How ironic.

I remember the most frustrating thing was that I could not give anyone or even myself the answer as to what caused me to experience such excruciating back pain. The lumbar MRI I had at that time showed some mild disc degeneration at L4-L5 and L5-S1, but no herniation or stenosis. Most medical literature stated that disc degeneration did not cause pain as it existed in many people who were asymptomatic. I could not find any plausible explanation from all professional resources I consulted. At that time, at the age of 33, my low back pain made me seriously consider filing for disability. As a matter of fact, it was largely the devastating low back pain, the associated suffering, and the difficulties and frustration in searching for an effective treatment that led me to become a dedicated pain care provider.

The basic concept that Zheng and Chen propose is that, in diseases such as cancer or stroke, the disease 
mechanism is non-recurrent, and all accepted controls have been event-free from the beginning. The conventional causality inference framework imposed by a traditional case-control study is impractical or inappropriate for studying the etiology of low back pain (1). This is because when a patient experiences a low back-pain episode, she (or he) will eventually recover from it. The natural history of low back pain shows not only age dependence, but also a pattern of remission and recurrence which is different from other irreversible clinical outcomes such as cancer or stroke. Thus, the absence of a pain episode during the same time interval cannot be accepted as evidence that the patient has never had a back pain episode in the past (1). They propose that, to truly understand the root cause of low back pain, the most feasible and most appropriate approach is to quantify and correct for the recurrent rhythm of low back pain, rather than to rely on our constrained ability to ascertain incident events and even more elusive controls (1).

Zheng and Chen also propose that the estimated transition age at which intervertebral discs lose the growth potential and begin degenerating is 13.3 years (1), while the estimated disc degeneration rate is $0.0344 /$ year. This is both interesting and informative because in clinical practice clinicians tend to downplay the subjective pain complaints of any adolescent, judging that patients in this age group could not possibility experience "real" back pain because of disc degeneration shown on MRI. Recently, Urrutia and colleagues (6) conducted a study to determine the prevalence of degenerative changes in the lumbar spine of pediatric patients. They examined 103 patients (mean age 6.6 years) who were evaluated with abdominal and pelvic MRI for indications that were not spine related. Urrutia et al (4) concluded that incidental findings related to lumbar disc degeneration are very uncommon in the population studied, in contrast to findings described in adults or later in adolescence. Dimar and colleagues (7) reviewed charts of 1,877 patients ( $<21$ years old) and selected those with chronic low back pain for over 6 months for analyses. They found that, in contrast to the common belief that lumbosacral degenerative disc disease commonly exists only in an older population, it actually begins earlier in the population of patients they studied.

Encouragingly, research on degenerative disc disease has picked up some momentum over the past recent years. A PubMed keyword search using "degenerative disc disease" returned 6,561 entries, with over 2,000 entries published since 2013. I believe the novel work by Zheng and Chen is of seminal significance and will shine more light upon our understanding of the mystery of causality between low back pain and disc degeneration. Considering the enormous burden of low back pain on people's lives at a global scale, the significance of their research discovery is remarkable, and such a creative and diligent research effort is truly laudable.

Xiulu Ruan, MD

Adjunct Clinical Associate Professor of Anesthesia

Dept. of Anesthesiology

Louisiana State University Health Science Center

1542 Tulane Ave.

New Orleans, LA 70112

Email: xiuluruan@yahoo.com

\section{References}

1. Zheng C-J, Chen J. Disc degeneration implies low back pain. Theor Biol Med Model 2015; 12:24.

2. Boden SD, Davis DO, Dina TS, Patronas NJ, Wiesel SW. Abnormal magnetic-resonance scans of the lumbar spine in asymptomatic subjects. A prospective investigation. J Bone Jt Surg 1990; 72:403-8.

3. Jensen MC, Brant-Zawadzki MN, Obuchowski N, Modic MT, Malkasian D, Ross JS. Magnetic resonance imaging of the lumbar spine in people without back pain. N Engl J Med 1994; 331:69-73.

4. Weber KT, Jacobsen TD, Maidhof R, Vi- rojanapa J, Overby C, Bloom O, Quraishi S, Levine M, Chahine NO. Developments in intervertebral disc disease research: pathophysiology, mechanobiology, and therapeutics. Curr Rev Musculoskelet Med 2015; 8:18-31.

5. Vos T, Barber RM, Bell B, Bertozzi-Villa A, Biryukov S, Bolliger I, Charlson F, Davis A, Degenhardt L, Dicker D. Global, 7 regional, and national incidence, prevalence, and years lived with disability for 301 acute and chronic diseases and injuries in 188 countries, 1990-2013: a systematic analysis for the Global Burden of Disease Study 2013. The Lancet 2015; 386:743-80o.

6. Urrutia J, Zamora T, Prada C. The prevalence of degenerative or incidental findings in the lumbar spine of pediatric patients: a study using magnetic resonance imaging as a screening tool. Eur Spine ] 2016; 25:596-601.

7. Dimar JR, Glassman SD, Carreon LY. Juvenile degenerative disc disease: a report of 76 cases identified by magnetic resonance imaging. Spine ] 2007; 7:332-7. 